\title{
On universal minimal proximal flows of topological groups
}

\author{
Xiongping Dai \\ Department of Mathematics, Nanjing University, Nanjing 210093, People's Republic of China \\ Eli Glasner \\ Department of Mathematics, Tel Aviv University, Tel Aviv, Israel
}

\begin{abstract}
In this paper, we show that the action of a characteristically simple, non-extremely amenable (non-strongly amenable, non-amenable) group on its universal minimal (minimal proximal, minimal strongly proximal) flow is effective. We present necessary and sufficient conditions, for the action of a topological group with trivial center on its universal minimal proximal flow, to be effective. A theorem of Furstenberg about the isomorphism of the universal minimal proximal flows of a discrete group and its subgroups of finite index ([8, Theorem II.4.4]) is strengthened. Finally, for a pair of groups $H<G$ the same method is applied in order to extend the action of $H$ on its universal minimal proximal flow to an action of its commensurator group $\operatorname{Comm}_{G}(H)$.
\end{abstract}

Keywords: Universal minimal proximal flow $\cdot$ Effective action $\cdot$ Strong/extreme amenability 2010 MSC: 37B05

\section{Introduction}

By a topological group we mean a group $G$ endowed with a $\mathrm{T}_{2}$-topology such that the algebraic operation $(x, y) \mapsto x y^{-1}$ be continuous from $G \times G$ to $G$. Unless stated otherwise, a flow is a triple $(G, X, \pi)$, sometimes write $(G, X)$, where $X$ is a compact $\mathrm{T}_{2}$-space, $G$ is a topological group with the neutral element $e$, and where the action map $\pi: G \times X \rightarrow X$ is such that

- $\pi:(t, x) \mapsto t x$ is jointly continuous; $\pi_{t} \circ \pi_{s}=\pi_{t s} \forall s, t \in T$; and $\pi_{e}=i d_{X}$ the identity map of $X$ to itself.

A flow $(G, X, \pi)$ is called effective if $t x=x$ for all $x \in X$ implies $t=e$; and we say $(G, X, \pi)$ is free if $t \in T$ with $t \neq e$ implies $t x \neq x$ for every $x \in X$ (see e.g. [1, 10]). Given any $t \in T$ with $t \neq e$, we will say that $(G, X, \pi)$ is effective at $t$ if $\pi_{t} \neq i d_{X}$, and free at $t$ if $t x \neq x$ for every $x \in X$.

By $\operatorname{Aut}(G)$ we will denote the group of topological automorphisms of $G$ and we will write $\operatorname{Aut}(G) t=\{a(t) \mid a \in \operatorname{Aut}(G)\}$. Let $\mathfrak{C}(G)$ be the center of the group $G$; that is,

$$
\mathfrak{C}(G)=\{t \in G \mid \operatorname{tg}=g t \quad \forall g \in G\} .
$$

We will show that the groups $\operatorname{Aut}(G)$ and $\mathfrak{C}(G)$ are important for the dynamics of the universal flows with phase group $G$.

Email addresses: xpdai@nju.edu.cn (Xiongping Dai), glasner@math.tau.il (Eli Glasner) Preprint submitted to $x x x$ 
Definition 1.1. A topological group $G$ is said to be (topologically) characteristically simple if $\langle\operatorname{Aut}(G) t\rangle$ is dense in $G$ for all $t \in G$ with $t \neq e$, where $\langle\operatorname{Aut}(G) t\rangle$ is the subgroup generated algebraically by $\operatorname{Aut}(G) t$.

Of course every (topologically) simple group is (topologically) characteristically simple. But, for example, for the topological group of rational numbers $\mathbb{Q}$, equipped with the topology it inherits from $\mathbb{R}$, we have $\operatorname{Aut}(\mathbb{Q})=\mathbb{Q}^{*}=\{t \in \mathbb{Q} \mid t \neq 0\}$, so clearly this abelian group is characteristically simple. Similarly, the groups $\mathbb{R}^{d}, d \geq 2$ are obviously characteristically simple. In [3] the reader can find a classification of the locally compact, compactly generated, characteristically simple groups.

A flow $(G, X, \pi)$ is said to be minimal if and only if there is no proper invariant closed subset of $X$-that is, $\operatorname{cls}_{X} G x=X$ for all $x \in X$. Since G. Birkhoff 1927 [2], minimal flows play a central role in the theory of topological dynamical systems (cf. $[6,5,8,15,1]$ etc.). In this paper, we will be mainly concerned with the effectiveness of the universal minimal and the universal minimal (strongly) proximal flows of a general topological group $G$.

In Section 3 we show that the action of a characteristically simple, non-extremely amenable (non-strongly amenable, non-amenable) group on its universal minimal (minimal proximal, minimal strongly proximal) flow is effective.

In Section 4 we present necessary and sufficient conditions, for the action of a topological group with trivial center on its universal minimal proximal flow, to be effective.

In Section 5 we strengthen a theorem of Furstenberg about the isomorphism of the universal minimal (strongly) proximal flows of a discrete group $G$ and its subgroups of finite index.

Finally, in Section 6, for a pair of groups $H<G$ the same method is applied in order to extend the action of $H$ on its universal minimal proximal flow to an action of its commensurator group $\operatorname{Comm}_{G}(H)$.

\section{Statements of the main results}

\subsection{Universal minimal flows}

Let $G$ be any topological group. Recall that a minimal flow $(G, X, \pi)$ is called a universal minimal flow of $G$ if any minimal flow $(G, Y, \psi)$ is a factor of $(G, X, \pi)$; that is, there exists a (not necessarily unique) continuous map $h$ from $X$ onto $Y$ with $h(\pi(t, x))=\psi(t, h(x))$ for all $t \in G$ and $x \in X$, written as $(G, X, \pi) \stackrel{h}{\rightarrow}(G, Y, \psi)$. See, e.g., $[4,15,1]$.

(A) Given any topological group $G$, there exists a unique (up to isomorphism) universal minimal flow $(G, \mathrm{M}(G)$ ). (See, e.g., [4, Corollary 1 and Theorem 2], [1, Theorem 8.1], and [12].) This theorem will be generalized to topological semigroups; see Theorem 3.5.

(B) If $G$ is a locally compact group, then its universal minimal flow is free. (See [4, Theorem 3] and [1, Theorem 8.3] for $G$ a discrete group; and [15, Theorem 2.2.1] for any locally compact group. See also [14, Section 3.3].)

However, for non-locally compact groups the question whether the universal minimal flow is free, is an interesting one and has drawn a lot of attention in the last decade; see e.g. [14]. For example, when $G$ is extremely amenable (i.e., the universal minimal flow of $G$ is trivial with onepoint phase space; cf., e.g., [9]), then the universal minimal flow of $G$ is of course not effective.

In the literature $[4,1,15]$, the freeness is usually proven by using the $\beta$-compactification of $G$. Using a different approach, established originally for proving the effectiveness of the universal minimal proximal flow in [8], we will show in $\$ 3.1$ the following result. 
Theorem 2.1. Let $G$ be a topological group which is not extremely amenable; and let $(G, \mathrm{M}(G))$ be its universal minimal flow. Then $(G, \mathrm{M}(G))$ is effective at every $t \in G$ with $\operatorname{cls}_{G}\langle\operatorname{Aut}(G) t\rangle=G$.

In particular, if the canonical flow $\operatorname{Aut}(G) \times G \rightarrow G$ by $(a, t) \mapsto a(t)$ is transitive at $t \in G$, then either $G$ is extremely amenable or $(G, \mathrm{M}(G))$ is effective at $t$.

It is interesting to note that our effectiveness condition of the universal minimal flow of a topological group is in fact independent of the phase space.

Corollary 2.2. Let $G$ be a characteristically simple group which is not extremely amenable; then $G$ acts effectively on its universal minimal space $\mathrm{M}(G)$.

A semiflow is a triple $(T, X, \pi)$ where $X$ is a compact $\mathrm{T}_{2}$-space, $T$ a topological semigroup with a neutral element $e$ (a monoid), and the action map $\pi: G \times X \rightarrow X$ satisfies the properties

- $\pi:(t, x) \mapsto t x$ is jointly continuous; $\pi_{t} \circ \pi_{s}=\pi_{t s} \forall s, t \in T$; and

- $\pi_{e}=i d_{X}$ the identity map of $X$ to itself.

Let $(T, X, \pi)$ and $(T, Y, \psi)$ be two semiflows; a continuous surjection $X \stackrel{h}{\rightarrow} Y$ is called a homomorphism from $(T, X, \pi)$ onto $(T, Y, \psi)$, written as $(T, X, \pi) \stackrel{h}{\rightarrow}(T, Y, \psi)$, if $h(\pi(t, x))=\psi(t, h(x))$ for all $t \in T$ and $x \in X$.

The idea of Theorem 2.1 is also useful for universal minimal semiflows of characteristically simple semigroups (with the obvious definition); see Theorem 3.6, Theorem 3.7 and Corollary 3.8 in $\$ 3.2$.

Of course proving that a flow is free is a much stronger result than showing that it is effective; so in that respect our effectiveness results yield nothing new for locally compact groups. Nonetheless, we hope that for non-locally compact acting groups this approach will become useful.

\subsection{Universal minimal proximal flows}

A flow $(G, X, \pi)$ is called proximal if for any $x, y \in X$ there is a net $\left\{t_{n}\right\}$ in $G$ such that $\lim t_{n} x=\lim t_{n} y$. Recall that a minimal proximal flow of a topological group $G$ is universal if it has every minimal proximal flow with the same phase group $G$ as a factor (cf. [8, §II.4]).

(C) For every topological group $G$, there exists a unique (up to isomorphism) universal minimal proximal flow for it. We will denote this universal minimal proximal flow by $(G, \Pi(G))$. (See [8, Proposition II.4.2].)

We notice that a topological group $G$ is strongly amenable if and only if $\Pi(G)$ is a singleton (cf. [8, p. 22]). Abelian, and more generally nilpotent, groups are strongly amenable (cf. [8, Theorem II.3.4]).

Let $G$ be a topological group; we denote by $\operatorname{Homeo}(\Pi(G))$ the group of self homeomorphisms of $\Pi(G)$ in the sequel.

(D) There is a homomorphism $a \mapsto \hat{a}$ of $\operatorname{Aut}(G)$ into Homeo $(\Pi(G))$. The homeomorphism $\hat{a}$ satisfies the equation $\hat{a}(t x)=a(t) \hat{a}(x)$ for every $t \in G$ and $x \in \Pi(G)$. For each $t \in G$ it sends the inner-automorphism $\sigma_{t}: g \mapsto t g t^{-1}$ to the homeomorphism $\widehat{\sigma}_{t}: x \mapsto t x$ of $\Pi(G)$. The flow $(G, \Pi(G))$ is effective if and only if the map $t \mapsto \widehat{\sigma}_{t}$, from $G$ to $\operatorname{Homeo}(\Pi(G))$, is one-to-one.

(This is due to Furstenberg; see [8, Proposition II.4.3].) 
It is easy to check that if $(G, \Pi(G))$ is effective, then the universal minimal flow of $G$ is also effective. So we are now concerned with the effectiveness of the universal minimal proximal flow $(G, \Pi(G))$. Since a central element of $G$ must act as the identity on $\Pi(G)$ we have to assume that $G$ has a trivial center, $\mathfrak{C}(G)=\{e\}$. Also notice that $\mathfrak{C}(G)$ is the kernel of the homomorphism $t \mapsto \sigma_{t}$ from $G$ into $\operatorname{Aut}(G)$.

We can strengthen Furstenberg's result (D) as follows:

Theorem 2.3. Let $G$ be a topological group with $\mathfrak{C}(G)=\{e\}$. Then the following statements are pairwise equivalent.

(1) $(G, \Pi(G))$ is effective.

(2) The map $t \mapsto \widehat{\sigma_{t}}$, from $G$ to $\operatorname{Homeo}(\Pi(G))$, is one-to-one.

(3) $a \mapsto \hat{a}$ of $\operatorname{Aut}(G)$ to $\operatorname{Homeo}(\Pi(G))$ is one-to-one.

Hence if one of the above (1),(2), (3) holds, then the universal minimal flow of $G$ is effective.

We will prove this theorem in $\S 4$ following the framework established in [8, §II.4].

It follows from (D) ([8, Proposition II.4.3]) that the universal minimal proximal flow $(G, \Pi(G))$ can be extended to a flow $(\operatorname{Aut}(G), \Pi(G))$, with the discrete topology of $\operatorname{Aut}(G)$, as follows:

$$
\xi: \operatorname{Aut}(G) \times \Pi(G) \rightarrow \Pi(G) ; \quad(a, x) \mapsto \hat{a}(x) .
$$

However, usually in $(\operatorname{Aut}(G), \Pi(G))$, we can not take $\operatorname{Aut}(G)$ to be equipped with its natural compact-open topology.

A simple application of Theorem 2.3 is Corollary 4.3 which says that, for $G$ with trivial center, $(\operatorname{Aut}(G), \Pi(G))$ is effective if so is $(G, \Pi(G))$.

In particular, in a way similar to the situation in Theorem 2.1, we may consider the effectiveness of the universal minimal proximal flow of a characteristically simple group.

Theorem 2.4. Let $G$ be a topological group which is not strongly amenable and let $(G, \Pi(G))$ be its universal minimal proximal flow; then the action of $G$ is effective at every $t \in G$ with $\operatorname{cls}_{G}\langle\operatorname{Aut}(G) t\rangle=G$.

Corollary 2.5. Let $G$ be a characteristically simple group which is not strongly amenable; then $G$ acts effectively on $\Pi(G)$.

Recall that a topological group has the Rohlin property if it has a dense conjugacy class in $G \backslash\{e\}$, and the strong Rohlin property if it has a co-meager conjugacy class in $G \backslash\{e\}$ (cf. [11, Definition 3.3]). To illustrate the subject, we give here several examples of Polish (non-locally compact) groups that have the Rohlin property:

(1) The group $\operatorname{Aut}(X, \mathscr{X}, \mu)$ of measure-preserving automorphisms of a standard measure space $(X, \mathscr{X}, \mu)$ equipped with the (Polish) weak topology.

(2) The group $U(H)$ of unitary operators on a separable infinite-dimensional Hilbert space $H$ equipped with the strong operator topology.

(3) The group of homeomorphisms of the Cantor set and the group of homeomorphisms of the Hilbert cube $[-1,1]^{\mathbb{N}}$, equipped with the topology of uniform convergence.

Examples of groups with the strong Rohlin property are: 
(4) The group $S_{\infty}(\mathbb{N})$ of all permutations of a countable set with the topology of pointwise convergence;

(5) The group $H_{+}[0,1]$ of order preserving homeomorphisms of the unit interval;

(6) The group $H(X)$ of homeomorphisms of a Cantor set.

We will say that a topological group has the characteristic Rohlin property if it has a dense $\operatorname{Aut}(G)$ orbit in $G \backslash\{e\}$ (i.e. there is some $g \in G$ with $\operatorname{Aut}(G) g$ dense in in $G \backslash\{e\}$ ), and the characteristic strong Rohlin property if it has a co-meager $\operatorname{Aut}(G)$ orbit in $G \backslash\{e\}$.

Clearly, the (strong) Rohlin property implies the characteristic (strong) Rohlin property. However, they are conceptually different. For instance, the abelian group $\mathbb{Q}$ has the strong characteristic Rohlin property.

By Theorem 2.4, we can easily obtain the following:

Corollary 2.6. Let $G$ be a topological group which is not strongly amenable. If $G$ has the characteristic (strong) Rohlin property, then there is a (co-meager) dense set $E$ of $G \backslash\{e\}$ such that $G$ acts effectively on $\Pi(G)$ at each $t \in E$.

See Corollary 4.6 for a related freeness criterion.

\subsection{An isomorphism theorem of universal minimal proximal flows}

The following result strengthens a theorem of Furstenberg (see [8, Theorem II.4.4]).

Theorem 2.7. Let $G$ be a topological group and $S$ a closed subgroup of finite index in $G$. Then $(S, \Pi(S))$ can be extended to $(G, \Pi(S))$ so that the flows $(G, \Pi(G))$ and $(G, \Pi(S))$ are isomorphic. Also, $(G, \Pi(G))$ is free if and only if so is $(G, \Pi(S))$.

We will prove this theorem in $\S 5$ by making use of some arguments established in [8].

\subsection{Universal minimal strongly proximal flows}

If a topological group $G$ acts on $Q$ which is a compact convex subset of a locally convex topological vector space and if each $t \in G$ acts as an affine transformation, then we say that $(G, Q)$ is an affine flow. See $[8,1]$.

Let $\mathcal{M}(X)$ be the set of all quasi-regular Borel probability measures on the compact $\mathrm{T}_{2}$ space $X$, which is compact and convex under the weak-* topology. Given any flow $(T, X, \pi)$, let $\left(T, \mathcal{M}(X), \pi_{*}\right)$ be the naturally induced affine flow on $\mathcal{M}(X)$. See [8, p. 31].

A flow $(T, X, \pi)$ is said to be strongly proximal if the induced affine flow $\left(T, \mathcal{M}(X), \pi_{*}\right)$ is proximal [7, p. 161] and [8, p. 31]. It is easy to see that a subflow of a strongly proximal flow is strongly proximal and every strongly proximal flow is also proximal. As observed in [8, p. 32] the diagonal-wise product of strongly proximal flows is strongly proximal. Here is a precise statement and a short proof:

(E) Strong proximality is preserved under diagonal-wise product of any cardinality.

Proof. Let $\left\{\left(G, X_{i}\right)\right\}_{i \in I}$ be a family of strongly proximal flows and set $X=\prod_{i \in I} X_{i}$ and $\operatorname{Pr}_{i}: x=\left(x_{i}\right)_{i \in I} \mapsto x_{i}$. Let $\mathfrak{m} \in \mathcal{M}(X)$ be any Borel probability measure. Then by [8, Lemma III.1.1], we may suppose that $m$ belongs to a minimal set $M$ of $(G, \mathcal{M}(X))$. Since $\operatorname{Pr}_{i}(M)$ is minimal for $\left(G, \mathcal{M}\left(X_{i}\right)\right)$ and $\left(G, \mathcal{M}\left(X_{i}\right)\right)$ is proximal, it follows that $\operatorname{Pr}_{i}(M) \subseteq X_{i}$ for all $i \in I$. This implies that $\operatorname{Pr}_{i}(\mathrm{~m})$ is a point mass for any $i \in I$ and thus $m$ itself is a point mass. This proves the statement. 
Hence as in $(\mathrm{C})$ before one proves that

(F) Associated to any topological group $G$, there exists a unique, up to an isomorphism, universal minimal strongly proximal flow. We will denote this flow by $\left(G, \Pi_{s}(G)\right)$. See $[8$, p. 32].

In the same way, Theorem 2.3 and Theorem 2.7 can be restated for $\Pi_{s}$ instead of $\Pi$. Moreover, by using the characterizations of amenable group given in [8, Theorem III.3.1], we can restate Theorem 2.4, Corollary 2.5 and Corollary 2.6, respectively, as follows.

Theorem 2.8. Let $G$ be a topological group which is not amenable and let $\left(G, \Pi_{s}(G)\right)$ be its universal minimal strongly proximal flow. Then $G$ acts effectively on $\Pi_{s}(G)$ at every $t \in G$ with $\operatorname{cls}_{G}\langle\operatorname{Aut}(G) t\rangle=G$.

Corollary 2.9. Let $G$ be a characteristically simple group which is not amenable; then $G$ acts effectively on $\Pi_{s}(G)$.

Corollary 2.10. Let $G$ be a topological group which is not amenable. If $G$ has the characteristic (strong) Rohlin property, then there is a (co-meager) dense set $E$ of $G \backslash\{e\}$ such that $G$ acts effectively on $\Pi_{s}(G)$ at each $t \in E$.

In the following table we collect the corollaries 2.2, 2.5 and 2.9, all of which hold under the assumption that $G$ is characteristically simple.

\begin{tabular}{|l|l|l|}
\hline $\mathrm{M}(G) \neq p t$ & $G$ is not extremely amenable & $G$ is effective on $\mathrm{M}(G)$ \\
\hline$\Pi(G) \neq p t$ & $G$ is not strongly amenable & $G$ is effective on $\Pi(G)$ \\
\hline$\Pi_{s}(G) \neq p t$ & $G$ is not amenable & $G$ is effective on $\Pi_{s}(G)$ \\
\hline
\end{tabular}

Table 1: Effective actions of a characteristically simple group

\subsection{Universal irreducible affine flows}

We now consider the affine flow $\left(G, \mathcal{M}\left(\Pi_{s}(G)\right)\right)$ induced on the compact convex set $\mathcal{M}\left(\Pi_{s}(G)\right)$ of quasi-regular Borel probability measures on the universal minimal strongly proximal flow $\left(G, \Pi_{s}(G)\right)$. Recall that an affine flow $(G, Q)$ is irreducible if it contains no proper non-empty closed convex invariant subset [8].

Recall that an irreducible affine flow $(G, Q)$ is called a universal irreducible affine flow of $G$ if for every irreducible affine flow $\left(T, Q^{\prime}\right)$ there exists an affine homomorphism $(G, Q) \stackrel{\phi}{\rightarrow}\left(G, Q^{\prime}\right)$.

(G) For any topological group $G,\left(G, \mathcal{M}\left(\Pi_{s}(G)\right)\right)$ is the (unique) universal irreducible affine flow. It is strongly proximal and contains $\Pi_{s}(G)$ (identified with the collection of Dirac measures, or equivalently the closed set of its extremal points) as its unique minimal set See [8, Proposition III.2.4].

Denote by AHomeo $\left(\mathcal{M}\left(\Pi_{s}(G)\right)\right)$ the group of all affine homeomorphisms of $\mathcal{M}\left(\Pi_{s}(G)\right)$ onto itself. In view of this result and because an action on $\Pi_{s}(G)$ determines the action on $\mathcal{M}\left(\Pi_{s}(G)\right)$ we immediately deduce the following: 
Theorem 2.11. There is a homomorphism $a \mapsto \hat{a}$ of $\operatorname{Aut}(G)$ into $\operatorname{AHomeo}\left(\mathcal{M}\left(\Pi_{s}(G)\right)\right)$ which, for $t \in G$, sends the inner-automorphism $\sigma_{t}: g \mapsto t g t^{-1}$ to the affine homeomorphism $\widehat{\sigma}_{t}: x \mapsto t x$ of $\mathcal{M}\left(\Pi_{s}(G)\right)$. The flow $\left(G, \mathcal{M}\left(\Pi_{s}(G)\right)\right)$ is effective if and only if the homomorphism

$$
t \mapsto \widehat{\sigma}_{t}, \quad G \rightarrow \operatorname{AHomeo}\left(\mathcal{M}\left(\Pi_{s}(G)\right)\right),
$$

is one-to-one.

Theorem 2.12. Let $G$ be a topological group and $S$ a closed subgroup of finite index in $G$. Then $\left(S, \mathcal{M}\left(\Pi_{s}(S)\right)\right)$ can be extended to $\left(G, \mathcal{M}\left(\Pi_{s}(S)\right)\right)$ so that the flows $\left(G, \mathcal{M}\left(\Pi_{s}(G)\right)\right)$ and $\left(G, \mathcal{M}\left(\Pi_{s}(S)\right)\right)$ are isomorphic.

\section{The effectiveness of some universal minimal flows}

This section is devoted to proving Theorem 2.1. Our argument is also useful for the effectiveness of the universal minimal semiflow associated to characteristically simple semigroups such as the additive semigroup $\mathbb{R}_{+}$with the usual Euclidean topology. See Theorem 3.6 below.

\subsection{Group actions}

Definition 3.1. A flow $(G, X, \pi)$ is said to be coalescent if every endomorphism of $(G, X, \pi)$ is an automorphism (cf., e.g., [1, p. 115]). Similarly one defines the notion of coalescence for semiflows.

The following result is due to Ellis (see [5]).

Lemma 3.2. The universal minimal flow of any topological group is coalescent.

Proof of Theorem 2.1. Let $(G, X, \pi)$ be the universal minimal flow of the topological group $G$ where $X$ is not one-point, namely, with $G$ not extremely amenable.

Write $\pi(t, x)=t x$ for all $t \in G$ and $x \in X$. Let $\operatorname{Homeo}(X)$ be the group of homeomorphisms of $X$ onto itself. For each $a \in \operatorname{Aut}(G)$ define a flow $\alpha: G \times X \rightarrow X$ by $(t, x) \mapsto t \cdot{ }_{a} x$, where

$$
t \cdot{ }_{a} x=a(t) x \quad \forall t \in G \text { and } x \in X .
$$

Since $a(G)=G$, it follows that $(G, X, \alpha)$ is minimal and by the university of $(G, X, \pi)$ there is a homomorphism $(G, X, \pi) \stackrel{\hat{a}}{\rightarrow}(G, X, \alpha)$. Clearly, $\hat{a}(t x)=a(t) \hat{a}(x)$ for every $t \in G$ and $x \in X$.

Now for $a^{-1}$ in place of $a \in \operatorname{Aut}(G)$, define similarly a minimal flow $\left(G, X, \alpha^{-1}\right)$ and to obtain another homomorphism $(G, X, \pi) \stackrel{\widehat{a^{-1}}}{\longrightarrow}\left(G, X, \alpha^{-1}\right)$ such that

$$
\widehat{a^{-1}}(t x)=a^{-1}(t) \widehat{a^{-1}}(x)
$$

for every $t \in G$ and $x \in X$.

Now, the composition map $\hat{a} \circ \widehat{a^{-1}}$ satisfies

$$
\hat{a} \circ \widehat{a^{-1}}(t x)=\hat{a}\left(a^{-1}(t) \widehat{a^{-1}}(x)\right)=a\left(a^{-1}(t)\right) \hat{a}\left(\widehat{a^{-1}}(x)\right)=t \hat{a} \circ \widehat{a^{-1}}(x),
$$

so that $\hat{a} \circ \widehat{a^{-1}}$ is an endomorphism of $(G, X, \pi)$. By Lemma 3.2, it follows that $\hat{a} \in \operatorname{Homeo}(X)$ for each $a \in \operatorname{Aut}(G)$. 
Next, arguing by contradiction, assume that for some $t \in G$ with $\operatorname{cls}_{G}\langle\operatorname{Aut}(G) t\rangle=G, t x=x$ for all $x \in X$. Then by the following commutative diagram:

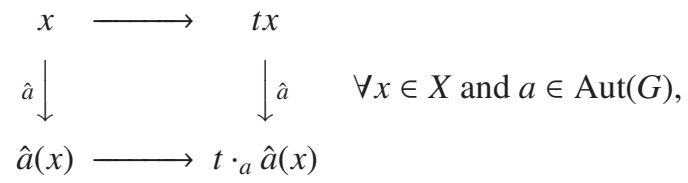

it follows that

$$
\hat{a}(t x)=a(t) \hat{a}(x)=\hat{a}(x) \quad \forall x \in X \text { and } a \in \operatorname{Aut}(G) .
$$

Since $\hat{a} \in \operatorname{Homeo}(X)$, it follows that $a(t) y=y$ for every $y \in X$. Then, for any $n \geq 1$

$$
a_{1}(t) \cdots a_{n}(t) y=y \quad \forall y \in X \text { and } a_{1}, \ldots, a_{n} \in \operatorname{Aut}(G) .
$$

Thus by $\operatorname{cls}_{G}\langle\operatorname{Aut}(G) t\rangle=G$, it follows that $G y=y$ and by minimality $X=\{y\}$. This contradicts the hypothesis that $X$ is not a singleton.

The proof of Theorem 2.1 is therefore completed.

Theorem 3.3. Let $G$ be a topological group such that $\operatorname{Aut}(G) t$ is dense in $G \backslash\{e\}$ for all $t \in G$ with $t \neq e$. If $(G, \mathrm{M}(G))$ is free at some element $\tau \in G$, then $(G, \mathrm{M}(G))$ is free.

Proof. Let $t$ be an arbitrary element of $G$ with $t \neq e$. By contradiction, assume that $t x_{0}=x_{0}$ for some $x_{0} \in \mathrm{M}(G)$. Since $\operatorname{Aut}(G) t$ is dense in $G \backslash\{e\}$, we can choose a net $\left\{a_{n}\right\}$ in $\operatorname{Aut}(G)$ such that $a_{n}(t) \rightarrow \tau$ and $a_{n}(t) \hat{a}_{n}\left(x_{0}\right)=\hat{a}_{n}\left(x_{0}\right)$. Since $\mathrm{M}(G)$ is compact, then, passing to a subnet if necessary, we may assume $\hat{a}_{n}\left(x_{0}\right) \rightarrow y$ in $\mathrm{M}(G)$. Thus $\tau y=y$, which contradicts the assumption that $(G, \mathrm{M}(G))$ is free at $\tau$.

\subsection{Universal minimal semiflows and effectiveness}

Given any topological semigroup $T$, there exists a unique (up to isomorphism) universal minimal semiflow of $T$, written as $(T, \mathrm{M}(T))$, as in the group case, such that if $(T, X)$ is a minimal semiflow there is a homomorphism $(T, \mathrm{M}(T)) \stackrel{\phi}{\rightarrow}(T, X)$. For this we need the semigroup version of Lemma 3.2.

Lemma 3.4. Let $(T, X, \pi)$ be a semiflow with $T$ a topological semigroup. Then there is a cardinal number a and a minimal subset $M$ of $\left(T, X^{\mathrm{a}}, \pi^{\mathrm{a}}\right)$ such that $\left(T, M, \pi^{\mathrm{a}}\right)$ is coalescent.

Proof. According to Zorn's lemma, let $C$ be a maximal almost periodic set of $(T, X)$; that is, for any finite subset $\left\{x_{1}, \ldots, x_{n}\right\} \subseteq C$, the point $\left(x_{1}, \ldots, x_{n}\right)$ is an almost periodic point for the diagonal-wise product semiflow $\left(T, X^{n}\right)$; and no other almost periodic set of $(T, X)$ properly contains it (cf. [1, p. 67]).

Let $z \in X^{C}$ such that range $z=C$ and $z: C \rightarrow X$ is one to one (for example, $z_{c}=c$, for each $c \in C)$. Let $M=\operatorname{cls}_{X^{C}} T z$, and let $z^{\prime} \in M$. Now $z^{\prime}$ is an almost periodic point for $\left(T, X^{C}\right)$, and so $C^{\prime}=$ range $z^{\prime}$ is an almost periodic set of $(T, X)$. In fact, it is easy to verify that $C^{\prime}$ is also maximal, and $z^{\prime}: C \rightarrow C^{\prime}$ is one to one.

Now, let $\varphi$ be an endomorphism of the minimal semiflow $(T, M)$. Then $(z, \varphi(z))$ is an almost periodic point of $(T, M \times M)$, so range $z \cup \operatorname{range} \varphi(z)$ is an almost periodic set of $(T, X)$. But range $z$ and range $\varphi(z)$ are both maximal almost periodic sets of $(T, X)$, so range $z=\operatorname{range} \varphi(z)$. 
If $\gamma$ is a permutation (bijection) of $C$, let $\gamma^{*}$ denote the induced automorphism of $\left(T, X^{C}\right)$ by $\gamma^{*}(z)_{c}=z_{\gamma(c)}$ for each $c \in C$. Define $\gamma$ by $z_{\gamma(c)}=\varphi(z)_{c}$, for $c \in C$. Since $\varphi(z)$, (regarded as a map of $C$ to $X)$ is one to one and range $\varphi(z)=$ range $z, \gamma$ is a permutation of $C$ and $\gamma^{*}(z)=\varphi(z)$.

Since $\gamma^{*}=\varphi$ restricted to $T z$ and $\operatorname{cls}_{X} T z=M$, then $\gamma^{*}=\varphi$ on $M$ and so $\varphi$ is an automorphism.

Now we can obtain the unique universal minimal semiflow of a topological semigroup following the framework of the proofs in [5], [8, Proposition II.4.2] and [1, Theorem 8.1].

Theorem 3.5. For any topological semigroup $T$, there exists a universal minimal semiflow $(T, \mathrm{M}(T), \pi)$, and any two universal minimal semiflows for $T$ are isomorphic.

Proof. Let $\mathscr{M}=\left\{\left(T, X_{i}, \pi_{i}\right) \mid i \in I\right\}$ be the collection of non-isomorphic minimal semiflows with the phase semigroup $T$. Define

$$
X=\prod_{i \in I} X_{i} \quad \text { and } \quad \pi:\left(t,\left(x_{i}\right)_{i \in I}\right) \mapsto\left(t x_{i}\right)_{i \in I} \text { from } T \times X \text { to } X .
$$

By Lemma 3.4 there is a cardinal number a and a minimal subset $M$ of $\left(T, X^{\mathrm{a}}, \pi^{\mathrm{a}}\right)$ such that $\left(T, M, \pi^{\mathrm{a}}\right)$ is coalescent. Obviously $\left(T, M, \pi^{\mathrm{a}}\right)$ is a universal minimal semiflow of $T$. Suppose $\left(T, Z, \pi_{\mathrm{Z}}\right)$ is another universal minimal semiflow of $T$. Then there are $T$-homomorphisms

$$
\left(T, M, \pi^{\mathrm{a}}\right) \stackrel{\phi}{\rightarrow}\left(T, Z, \pi_{\mathrm{Z}}\right) \stackrel{\psi}{\rightarrow}\left(T, M, \pi^{\mathrm{a}}\right) .
$$

Since $\left(T, M, \pi^{\mathrm{a}}\right)$ is coalescent, $\psi \circ \phi$ and also $\phi, \psi$ are all isomorphisms.

Next we will be concerned with the effectiveness of the universal minimal semiflow of some topological semigroups including $\mathbb{R}_{+}=[0,+\infty)$ or $\mathbb{Q}_{+}=\mathbb{Q} \cap[0, \infty)$ Of course for $\mathbb{R}_{+}$this way of proving effectiveness is an overkill, as already the action of $\mathbb{R}_{+}$on the 2 -torus via an irrationally oriented line is minimal and effective (hence free). However, for general acting non locally compact semigroup our next results may be of interest.

Let $T$ be a topological semigroup. By $\mathcal{E}$ nd $(T)$ we denote the set of continuous self homomorphisms $a$ of $T$ such that $a(T)$ is dense in $T$. By $(T, \mathrm{M}(T))$ we denote the universal minimal semiflow of $T$. It is easy to check that:

- Given any $t \in \mathbb{R}_{+}$with $t \neq 0, \quad \mathcal{E}$ nd $\left(\mathbb{R}_{+}\right) t=\left\{a(t) \mid a \in \mathcal{E}\right.$ nd $\left.\left(\mathbb{R}_{+}\right)\right\}=(0,+\infty)$.

By a slight modification of the proof of Theorem 2.1 we can obtain the following:

Theorem 3.6. Let $T$ be a topological semigroup such that $\langle\mathcal{E}$ nd $(T) t\rangle$ is dense in $T$ for all $t \in T$ with $t \neq e$. Then, either $T$ is extremely amenable or $(T, \mathrm{M}(T)$ ) is effective (i.e., $t: x \mapsto t x$ is not the identity map for any $t \in T$ with $t \neq e$ ).

It should be noted that even if $T$ is locally compact, Theorem 3.6 is already beyond the framework of Veech [15, Theorem 2.2.1] which is proven only for locally compact groups.

We say that a semiflow $(T, X, \pi)$ is free at $t \in T$ if $t x \neq x \forall x \in X$. We now have a semigroup version of Theorem 3.3:

Theorem 3.7. Let $T$ be a topological semigroup such that $\mathcal{E}$ nd $(T) t$ is dense in $T \backslash\{e\}$ for all $t \in T$ with $t \neq e$. If $(T, \mathrm{M}(T))$ is free at some element $\tau \in T$, then $(T, \mathrm{M}(T))$ is free. 
Corollary 3.8. Let $T$ be an abelian semigroup such that $\mathcal{E}$ nd $(T) t$ is dense in $T \backslash\{e\}$ for all $t \in T$ with $t \neq e$. Then $(T, \mathrm{M}(T))$ is free if $T$ is not extremely amenable.

Proof. If $(T, \mathrm{M}(T))$ is free at some $\tau \in T$, then by Theorem 3.7 it is free. Now let there be some $\tau \in T$ with $\tau \neq e$ such that $\tau x_{0}=x_{0}$ for some point $x_{0} \in X$. Then $\tau t x_{0}=t x_{0}$ for any $t \in T$. Since $T x_{0}$ is dense in $X$ by minimality, hence $\tau x=x$ for all $x \in X$. However, this contradicts Theorem 3.6. Thus $(T, \mathrm{M}(T))$ is free.

\section{The effectiveness of some universal minimal proximal flows}

This section will be devoted to proving Theorems 2.3 and 2.4. Let $G$ be a topological group. First, we will need a lemma.

Lemma 4.1 (See [8, Lemma II.4.1]). The only endomorphism a minimal proximal G-flow admits is the identity automorphism.

In fact we can obtain a more general uniqueness result.

Lemma 4.2. If $\left(G, X, \pi_{X}\right) \stackrel{\theta}{\rightarrow}\left(G, Y, \pi_{Y}\right)$ is a homomorphism (not necessarily surjective) from a minimal proximal flow $\left(G, X, \pi_{X}\right)$ to another proximal flow $\left(G, Y, \pi_{Y}\right)$, then $\theta$ is the unique homomorphism admitted from $\left(G, X, \pi_{X}\right)$ to $\left(G, Y, \pi_{Y}\right)$.

Proof. Let $\left(G, X, \pi_{X}\right) \stackrel{\phi}{\rightarrow}\left(G, Y, \pi_{Y}\right)$ be a homomorphism. Then given any $x \in X$, set $y_{1}=\theta(x)$ and $y_{2}=\phi(x)$. By proximality there is a net $\left\{t_{n}\right\}$ in $G$ and some $y_{\infty} \in Y$ such that

$$
\lim _{n} t_{n} y_{1}=\lim _{n} t_{n} y_{2}=y_{\infty} .
$$

We can assume that the limit, $x_{\infty}=\lim t_{n} x$ exists and then $\theta\left(x_{\infty}\right)=y_{\infty}=\phi\left(x_{\infty}\right)$. Since $(G, X)$ is minimal, we conclude that $\theta(x)=\phi(x)$. Thus $\theta=\phi$ on $X$.

Recall that $\sigma_{t}: s \mapsto t s t^{-1}$ is the inner-automorphism of $G$ as in (D) in $\S 2.2$. Let $(G, \Pi(G), \pi)$ be the universal minimal proximal flow associated with $G$ and simply write $\pi(t, x)=t x$ for $t \in G$ and $x \in \Pi(G)$ as in (C) in $\S 2.2$. We also recall the construction of the homomorphism $a \mapsto \hat{a}$ of $\operatorname{Aut}(G)$ to Homeo( $\Pi(G))$ introduced in [8, p. 23]. In fact, this is in essence the same as the construction described above for $\mathrm{M}(G)$ in the proof of Theorem 2.1; the only difference is that here we use the fact that $\Pi(G)$ admits no non-trivial endomorphisms instead of the coalescence of $\mathrm{M}(G)$.

It should be mentioned that usually we cannot expect the continuity of the homomorphism $a \mapsto \hat{a}$, from $\operatorname{Aut}(G)$ to $\operatorname{Homeo}(\Pi(G))$, when the former is equipped with its natural compactopen or pointwise convergence topologies.

Proof of Theorem 2.3. (1) $\Leftrightarrow$ (2): First note that the map $t \mapsto \widehat{\sigma}_{t}$ from $G$ to $\operatorname{Homeo}(\Pi(G))$ is a homomorphism. Then the statement easily follows from $\widehat{\sigma}_{t}=t$.

$(1) \Rightarrow(3)$ : Let $a \in \operatorname{Aut}(G)$ be such that $\hat{a}$ is the identity map on $\Pi(G)$. Then by the equality $\hat{a}(t x)=a(t) \hat{a}(x)$, it follows that $t x=a(t) x$ for all $x \in X$ and $t \in G$. Since $(G, \Pi(G), \pi)$ is effective, then $a(t)=t$ for every $t \in G$ and so $a=i d_{G}$. This shows that the homomorphism $a \mapsto \hat{a}$ is one-to-one.

(3) $\Rightarrow(2)$ : Since $\mathfrak{C}(G)=\{e\}$, the map $t \mapsto \sigma_{t}$ from $G$ to $\operatorname{Aut}(G)$ is one-to-one. Thus by condition (3), it follows that $t \mapsto \widehat{\sigma}_{t}$ is one-to-one.

The proof of Theorem 2.3 is completed. 
Proof of Theorem 2.4. This proof is analogous to the proof of Theorem 2.1 and so we omit the details.

Let $(\operatorname{Aut}(G), \Pi(G))$ be the canonical extension of $(G, \Pi(G))$. Then we can easily obtain the following by Theorem 2.3 .

Corollary 4.3. Let $G$ be a topological group with $\mathfrak{C}(G)=\{e\}$. Then $(G, \Pi(G))$ is effective if and only if so is $(\operatorname{Aut}(G), \Pi(G))$.

In many cases the group of inner-automorphisms $\operatorname{Inn}(G)=\left\{\sigma_{t} \mid t \in G\right\}$ is a proper subgroup of $\operatorname{Aut}(G)$ and so Corollary 4.3 seems to be interesting. The "if" part of Corollary 4.3 will be generalized in Theorem 4.4 below.

Next we consider the inheritance of freeness of the universal minimal flows associated to topological semigroups.

Theorem 4.4. Let $T$ be a topological semigroup and $H$ a subsemigroup of $T$. Then

(1) If $(T, \mathrm{M}(T))$ is free, then $(H, \mathrm{M}(H))$ is also free.

(2) If $(T, \Pi(T))$ is free, then $(H, \Pi(H))$ is also free.

(3) If $\left(T, \Pi_{s}(T)\right)$ is free, then $\left(H, \Pi_{s}(H)\right)$ is also free.

Proof. Let $(T, \mathrm{M}(T))$ be free; then $(H, \mathrm{M}(T))$ is also free. Now let $X$ be an $H$-minimal subset of $\mathrm{M}(T)$ and then $(H, X)$ is obviously free. Then by the universality of $(H, \mathrm{M}(H))$, it follows that there is a homomorphism $(H, \mathrm{M}(H)) \stackrel{\phi}{\rightarrow}(H, X)$. This implies that $(H, \mathrm{M}(H))$ is free. This proves (1). We can easily prove (2) and (3) similarly.

Theorem 4.5. Let $G$ be a topological group with $\operatorname{Aut}(G) t$ dense in $G \backslash\{e\}$ for some $t \in G$ with $t \neq e$. If $(G, \Pi(G))$ is free at some $\tau \in G$, then $(G, \Pi(G))$ is free at $t$. here.

The proof is almost verbatim the same as that of Theorem 3.3 and thus we omit its details

The following is a simple consequence of Theorem 4.5, which is comparable with Corollary 2.6.

Corollary 4.6. Let $G$ be a topological group with the characteristic (strong) Rohlin property, such that $(G, \Pi(G))$ is free at some $\tau \in G$. Then there exists a (co-meager) dense subset $E$ of $G \backslash\{e\}$ such that $G$ acts freely on $\Pi(G)$ at each $t \in E$.

We note that in the same way the above Corollary 4.3, Theorem 4.5 and Corollary 4.6 can be restated for $\Pi_{s}(G)$ in place of $\Pi(G)$.

\section{A generalization of Furstenberg's isomorphism theorem}

Based on the construction of the homomorphism $a \mapsto \hat{a}$ presented in $\S 3$, this section will be mainly devoted to proving Theorem 2.7. For that, let $G$ be a topological group and let $S$ be a closed proper subgroup of finite index in $G$, unless otherwise specified.

First of all, we will need a useful lemma.

Lemma 5.1 (See [8, Lemma II.3.2]). Let $T$ be a topological group and $(T, X, \varphi)$ a minimal proximal flow. 
1. If $T$ is a compact extension of its subgroup $L$, then $\left(L, X,\left.\varphi\right|_{L \times X}\right)$ is minimal and proximal.

2. If $L$ is a closed subgroup of finite index in $T$, then $\left(L, X,\left.\varphi\right|_{L \times X}\right)$ is minimal and proximal.

Here $T$ is called a compact extension of $L$ if $L$ is a closed normal subgroup of $T$ such that the quotient group $T / L$ is a compact group.

We also will need a technical lemma.

Lemma 5.2. There exists a normal closed subgroup $N$ of $G$ such that $N \subseteq S$ and that $N$ is of finite index in $G$.

Proof. Under the discrete topology of $G, S$ contains a normal subgroup, say $M$, of $G$ of finite index in $G$ (see [13, p. 26] or [8, p. 24]). Now let $N=\operatorname{cls}_{G} M$. Then it is easy to check that $N$ is a normal closed subgroup of $G$ satisfying the claim of the lemma.

From now on, let $N$ be as in Lemma 5.2. Let $(G, \Pi(G)),(S, \Pi(S))$ and $(N, \Pi(N))$ be the universal minimal proximal flows of the topological groups $G, S$ and $N$, respectively, as in (C) in $\S 2.2$.

First we need to extend the natural action of $N$ on $\Pi(N)$ to an action of $G$ on $\Pi(N)$. For this we define an action of $G$ on $\Pi(N)$ by mapping $G$ into $\operatorname{Aut}(N)$ as follows:

$$
\zeta: G \times \Pi(N) \rightarrow \Pi(N) ; \quad(t, z) \mapsto \widehat{\left.\sigma_{t}\right|_{N}}(z),
$$

where ${ }^{\wedge}: \operatorname{Aut}(N) \rightarrow \operatorname{Homeo}(\Pi(N))$ is the canonical map introduced in $\S 3$, with $N$ in place of $G$.

Lemma 5.3. $(G, \Pi(N), \zeta)$ is a flow, and as such it is minimal and proximal.

Proof. We only need to verify $\widehat{\left.\sigma_{t}\right|_{N}}(z)$ is jointly continuous with respect to $t \in G$ and $z \in \Pi(N)$. Since $N$ is closed and of finite index in $G$, there exists a finite set, say $\left\{s_{1}, \ldots, s_{k}\right\} \subseteq G$, such that $G=s_{1} N \cup \cdots \cup s_{k} N$ is a clopen partition and $s_{i} N \cap s_{j} N=\emptyset$ for $1 \leq i \neq j \leq k$. Now suppose that the net $\left(t_{i}, z_{i}\right) \rightarrow(t, z)$ in $G \times \Pi(N)$. Then, passing to subnets (and relabeling) we can assume that for some fixed $j_{0}$ we have $t_{i} \in s_{j_{0}} N$ for every $i$. Thus for every $i$ there is some $r_{i} \in N$ so that $t_{i}=s_{j_{0}} r_{i}$ and $r_{i} \rightarrow r:=s_{j_{0}}^{-1} t$. Observe that for $r \in N$ and $z \in \Pi(N)$ we have $\widehat{\sigma_{r} \Gamma_{N}}(z)=r z$. Now, by the continuity of the $N$ action on $\Pi(N)$,

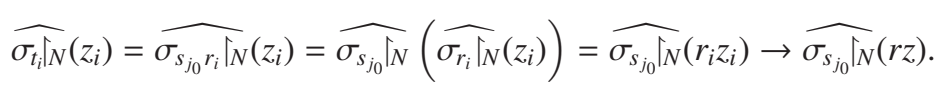

But

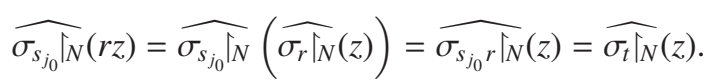

Thus $\zeta: G \times \Pi(N) \rightarrow \Pi(N)$ is continuous.

Remark 5.4. One can relax here the assumption that $N$ has finite index in $G$. Assuming only that $N$ is a clopen normal subgroup will suffice. Indeed, under this assumption the space $G / N$ is discrete and (denoting the quotient map $Q: G \rightarrow G / N$ ) we have, in the notation of the proof above, $Q\left(t_{i}\right) \rightarrow Q(t)$. Thus, eventually $Q\left(t_{i}\right)=Q(t)$ and we can assume that for every $i, t_{i}=s_{j_{0}} r_{i}$ for some fixes $s_{j_{0}} \in G$ and $r_{i} \in N$. Now proceed as before.

With the above preparations at hand, we are ready to complete our proof of Theorem 2.7. 
Proof of Theorem 2.7. By the universality of $(G, \Pi(G))$, there exists a $(G, \Pi(G)) \stackrel{\phi}{\rightarrow}(G, \Pi(N))$, where $(G, \Pi(N))=(G, \Pi(N), \zeta)$ as in Lemma 5.3.

Next consider the flow $(N, \Pi(G))$ which is obtained by restricting the action of $G$ on $\Pi(G)$ to the action of its subgroup $N$. By Lemma 5.1 this flow is minimal and proximal; therefore there is a homomorphism $(N, \Pi(N)) \stackrel{\psi}{\rightarrow}(N, \Pi(G))$. Now $(N, \Pi(N)) \stackrel{\phi \circ \psi}{\longrightarrow}(N, \Pi(N))$ is an endomorphism, hence it is the identity map by Lemma 4.1. Therefore $(G, \Pi(G)) \stackrel{\phi}{\rightarrow}(G, \Pi(N))$ is an isomorphism.

By Lemma 5.1 again, $(S, \Pi(G))$ is minimal and proximal, hence there exists a homomorphism $(S, \Pi(S)) \stackrel{\theta}{\rightarrow}(S, \Pi(G))$. Similarly, $(N, \Pi(S))$ is minimal and proximal and there exists a homomorphism $(N, \Pi(N)) \stackrel{\lambda}{\rightarrow}(N, \Pi(S))$. Thus the composition

$$
(N, \Pi(N)) \stackrel{\lambda}{\rightarrow}(N, \Pi(S)) \stackrel{\theta}{\rightarrow}(N, \Pi(G)) \stackrel{\phi}{\rightarrow}(N, \Pi(N))
$$

is an endomorphism of $(N, \Pi(N))$, hence it is the identity map. Thus $(N, \Pi(N)) \stackrel{\lambda}{\rightarrow}(N, \Pi(S))$ is an isomorphism. Using this isomorphism, together with the isomorphism $(G, \Pi(G)) \stackrel{\phi}{\rightarrow}(G, \Pi(N))$, an action of $G$ on $\Pi(S)$ can be defined so that $(G, \Pi(S))$ and $(G, \Pi(G))$ are isomorphic.

It is well known that for locally compact groups every subgroup of an amenable group is amenable. How about strongly amenable groups?

Corollary 5.5. Let $G$ be a topological group and $S$ a closed subgroup of finite index in $G$. Then $G$ is strongly amenable if and only if so is $S$.

Proof. By Theorem 2.7, it follows that $\Pi(G) \cong \Pi(S)$. So $\Pi(G)$ is a singleton if and only if so is $\Pi(S)$. This proves Corollary 5.5.

Question 5.6. Let $G$ be a locally compact group. If $G$ is a compact extension of $S$, is it true that $(G, \Pi(G)) \cong(G, \Pi(S))$ ?

If the answer to Question 5.6 is positive, then as in Corollary 5.5 we will conclude that $G$ is strongly amenable if and only if so is $S$.

\section{Commensurators}

If $G$ is a group and $H<G$ a subgroup, we denote $H^{g}=g H g^{-1}$ and $H_{g}=H \cap H^{g}$. The commensurator of $H$ in $G$ is defined by

$$
\boldsymbol{H}=\operatorname{Comm}_{G}(H)=\left\{g \in G \mid H_{g} \text { has finite index in both } H \text { and } H^{g}\right\} .
$$

As a corollary of [8, Theorem II.4.4] we obtain the following.

Theorem 6.1. Assume that $\boldsymbol{H}$ is countable. Then, the canonical action of $H$ on $\Pi(H)$ can be extended to an action $(\boldsymbol{H}, \Pi(H))$. That is, there is a homomorphism $g \mapsto \hat{g}$, from $\boldsymbol{H}$ to Homeo(П $(H))$, such that

- $\hat{g}$ satisfies the equation $\hat{g}(t x)=\left(g g^{-1}\right) \hat{g}(x)$ for every $t \in H \cap g^{-1} H g$ and $x \in \Pi(H)$.

- for $h \in H$ and $x \in \Pi(H)$ we have $\hat{h}(x)=h(x)$. 
- for every $g \in \boldsymbol{H}$

$$
(H, \Pi(H)) \cong\left(H, \Pi\left(H_{g}\right)\right) \cong\left(H^{g}, \Pi\left(H_{g}\right)\right) \cong\left(H^{g}, \Pi\left(H^{g}\right)\right) .
$$

Analogous statements hold for $\Pi_{s}$.

Proof. Let $\left\{e=g_{0}, g_{1}, g_{2}, \ldots, g_{k}, \ldots\right\}$ be an enumeration of $\boldsymbol{H}$ and for each $k$ let $H_{k}=\bigcap_{i=0}^{k} g_{i} H^{g_{i}}$. Then, $H_{k}$ has finite index in $H$ and there is a normal subgroup $N$ of $H$, with $N<H_{k}$, such that $N$ is of finite index in $H$. The flow $(N, \Pi(H))$ is minimal, proximal and for each $0 \leq i \leq k$, the map $\sigma_{g_{i}}\left\lceil N\right.$ is an automorphism of $N$. Thus the corresponding map $\widehat{\sigma_{g_{i}}\lceil N}: \Pi(H) \rightarrow \Pi(H)$ is a homeomorphism and the map ${ }^{`}:\left\langle g_{0}, g_{1}, g_{2}, \ldots, g_{k}\right\rangle \rightarrow \operatorname{Homeo}(\Pi(H))$ is a group homomorphism. Note that when $N_{1}<N_{2}<H$ and $g \in \boldsymbol{H}$ normlizes both $N_{1}$ and $N_{2}$ we have, with $\widehat{g_{j}}$ the corresponding homeomorphisms induced by $\sigma_{g_{j}} \Gamma_{N_{j}}, j=1,2$,

$$
\widehat{g_{2}-1}\left(\widehat{g_{1}}(t x)\right)=\widehat{g_{2}^{-1}}\left(g^{-1} \widehat{g}^{-1}(x)\right)=\widehat{\operatorname{tg}_{2}^{-1}}\left(\widehat{g_{1}}(x)\right),
$$

for every $t \in N_{1}$. Thus $\phi=\widehat{g_{2}^{-1}} \circ \widehat{g_{1}}$ is an automorphism of the minimal proximal flow $\left(N_{1}, \Pi(H)\right)$, whence $\widehat{g_{2}-1} \circ \widehat{g_{1}}=i d_{\Pi(H)}$, and $\widehat{g_{2}}=\widehat{g_{1}}$.

We now let $k \rightarrow \infty$ to conclude the proof.

With $G=\operatorname{SL}(n, \mathbb{R})$ and $H=\operatorname{SL}(n, \mathbb{Z})$ we have $\operatorname{Comm}_{G}(H)=\operatorname{SL}(n, \mathbb{Q})$ and a nice instance of this theorem is the result that the action $(\operatorname{SL}(n, \mathbb{Z}), \Pi(\operatorname{SL}(n, \mathbb{Z})))$ extends to an action $(\operatorname{SL}(n, \mathbb{Q}), \Pi(\operatorname{SL}(n, \mathbb{Z})))$. Again an analogous result is valid for $\Pi_{s}$ instead of $\Pi$.

\section{Acknowledgments}

X. Dai was partly supported by National Natural Science Foundation of China (Grant Nos. 11431012, 11271183) and PAPD of Jiangsu Higher Education Institutions; and E. Glasner was supported by a grant of the Israel Science Foundation (ISF 668/13).

\section{References}

[1] J. Auslander, Minimal Flows and Their Extensions, North-Holland Math. Studies vol. 153. North-Holland, Amsterdam, 1988.

[2] G. Birkhoff, Dynamical Systems, Amer. Math. Soc. Colloq. Publ. vol. 9. AMS, Providence, R.I. 1927.

[3] P.-E. Caprace and M. Nicolas, Decomposing locally compact groups into simple pieces, Math. Proc. Cambridge Philos. Soc. 150 (2011), 97-128.

[4] R. Ellis, Universal minimal sets, Proc. Amer. Math. Soc. 11 (1960), 540-543.

[5] R. Ellis, Lectures on Topological Dynamics, W.A. Benjamin, Inc., 1969.

[6] H. Furstenberg, Disjointness in ergodic theory, minimal sets, and a problem in Diophantine approximation, Math. Systems Theory 1 (1967), 1-49.

[7] S. Glasner, Compressibility properties in topological dynamics, Amer. J. Math. 97 (1975), 148-171.

[8] E. Glasner, Proximal Flows, Lecture Notes in Math. 517, Springer-Verlag, 1976.

[9] E. Glasner, On minimal actions of Polish groups, Topology Appl. 85 (1998), 119-125.

[10] E. Glasner and V.V. Uspenskij, Effective minimal subflows of Bernoulli flows, Proc. Amer. Math. Soc. 137 (2009), 3147-3154.

[11] E. Glasner and B. Weiss, Topological groups with Rohlin properties, Colloq. Math. 110 (2008), 51-80.

[12] Y. Gutman and H. Li, A new short proof for the uniqueness of the universal minimal space, Proc. Amer. Math. Soc. 141 (2013), 265-267.

[13] E. Hewitt and K. Ross, Abstract Harmonic Analysis, Springer-Verlag, Berlin, 1963.

[14] V. Pestov, Dynamics of infinite-dimensional groups. The Ramsey-Dvoretzky-Milman phenomenon, University Lecture Series 40. American Mathematical Society, Providence, RI, 2006.

[15] W.A. Veech, Topological dynamics, Bull. Amer. Math. Soc. 83 (1977), 775-830. 